\title{
Vielfalt (er)leben und gestalten: Diversity- Schulung zur Sensibilisierung von Mitarbeitenden und zur nachhaltigen Etablierung diversitätsorientierter Arbeit im Bibliotheksalltag
}

Wie wird aus der institutionellen Diversity-Verpflichtung eine gemeinsame Zielsetzung, die auch die Mitarbeitenden teilen und verfolgen? Wie können Mitarbeitende zu Verbündeten werden, die Verantwortung für die Entwicklung und Umsetzung diversitätssensibler und diskriminierungskritischer Maßnahmen übernehmen und das Thema weiter vorantragen? In diesem Beitrag wird die Diversity-Schulungsreihe für Mitarbeitende der Stadtbibliothek Köln vorgestellt, die das Ziel verfolgt, das Thema Vielfalt in allen Arbeitsbereichen der Bibliothek nachhaltig zu etablieren. ${ }^{1}$ Sie wurde im Rahmen des Programms $360^{\circ}$ - Fonds für Kulturen der neuen Stadtgesellschaft zusammen mit der Berliner DiversityBeratungsorganisation Eine Welt der Vielfalt e. $V .^{2}$ konzipiert und durchgeführt.

\section{Hintergrund}

„Diversity“ bedeutet allgemein übersetzt Vielfalt bzw. Vielfältigkeit. Damit kann aber auch „Unterschiedlichkeit“ gemeint sein. Grundsätzlich sind nicht nur die Unterschiede, die alle Menschen haben, sondern immer auch die Gemeinsamkeiten gemeint.

Diversity als Konzept hat seinen Ursprung in den Bürgerinnenrechtsbewegungen diskriminierter Gruppen in den USA, die für eine gerechte Teilhabe kämpften (vgl. Abdul-Hussain \& Hofmann, 2013). Die rechtlichen Rahmenbedingungen für Diversity leiten sich aus internationalen Vereinbarungen, EUVorgaben und nationalen Gesetzen ab, zum Beispiel die Allgemeine Erklärung

1 Ich danke sehr herzlich meiner Kollegin Waltraud Reeder-Dertnig (die auch Teilnehmerin der Schulung war) für ihre hilfreichen Kommentare, Anregungen und inhaltlichen Ergänzungen.

2 Mehr zu Eine Welt der Vielfalt e. V. unter: https://www.ewdv-diversity.de/

๖ Open Access. (C 2021 Melisa Bel Adasme, publiziert von De Gruyter. (๕)BY Dieses Werk ist lizenziert unter der Creative Commons Attribution 4.0 Lizenz.

https://doi.org/10.1515/9783110726213-011 
der Menschenrechte ${ }^{3}$, das deutsche Grundgesetz $z^{4}$, das Allgemeine Gleichbehandlungsgesetz $(\mathrm{AGG})^{5}$ und das Landesantidiskriminierungsgesetz (LADG) Berlin ${ }^{6}$.

Die Kategorien, die durch das AGG vor Diskriminierung geschützt sind, können als Diversity-„Kerndimensionen“ bezeichnet werden. Diese machen in ihrer Verschränkung die Identität aus und markieren die (Nicht-)Zugehörigkeit zu bestimmten Gruppen. Sie sind die relativ unveränderbaren (und mehr oder weniger sichtbaren) Merkmale, aufgrund derer Menschen individuell, institutionell oder strukturell benachteiligt werden. Im gesellschaftlichen Kontext werden diese Merkmale unterschiedlich bewertet. Daraus ergibt sich zwischen ihnen eine hierarchische Beziehung, die mit Macht verbunden ist und $\mathrm{zu}$ (individuellen, institutionellen und/oder strukturellen) Mehrfachdiskriminierungen führen kann (vgl. Tanyllmaz \& Greve, 2018).

An dieser Stelle greift der Diversity-Ansatz mit seinem Verständnis und Zielen für öffentliche Einrichtungen: Es geht darum, Vielfalt und Differenz bewusst zu fördern, unterschiedliche Identitäten und Lebensformen anzuerkennen und wertzuschätzen, nicht auf Defizite zu achten, sondern Ressourcen hervorzuheben, eine ganz klare Antidiskriminierungshaltung einzunehmen sowie sich gesellschaftlicher, institutioneller und struktureller Barrieren bewusst zu werden, um diese abbauen zu können.

Öffentliche Bibliotheken verstehen sich als innovative Institutionen, die eine besondere Verantwortung gegenüber der sich wandelnden, diversen Gesellschaft tragen, in welche sie eingebettet sind. Sie haben den Anspruch, offen für alle Menschen zu sein und ihre Teilhabe zu fördern. Vor diesem Hintergrund gewinnt das Thema Diversity immer mehr an Bedeutung, vor allem in Hinblick auf eine zielgruppengerechte Ansprache und Angebotsgestaltung. Ein essenzieller und primärer Ansatzpunkt an dieser Stelle ist die diversitätsorientierte Sensibilisierung der Mitarbeitenden.

3 In Artikel 2 ist die Definition allgemeiner Rechte und Freiheiten ohne Unterschied nach diversen Kategorien wie zum Beispiel Hautfarbe, Geschlecht, Sprache, Religion enthalten (vgl. Generalversammlung der Vereinten Nationen, 1948).

4 Besonders in Artikel 3, Satz 2 und 3 wird die Verhinderung von Benachteiligung festgehalten (vgl. Bundesministerium der Justiz und für Verbraucherschutz).

5 Das regelt den Schutz vor Diskriminierung und Benachteiligung aus rassistischen Gründen oder wegen der ethnischen Herkunft, des Geschlechts, der Religion oder Weltanschauung, einer Behinderung, des Alters oder der sexuellen Identität durch private Akteur^innen (vgl. Antidiskriminierungsstelle des Bundes, 2020).

6 Das LADG ist das erste Gesetz dieser Art. Es zielt auf den Diskriminierungsschutz im öffentlich-rechtlichen Handeln der Verwaltung und öffentlicher Stellen und stellt einen erweiterten Katalog zu schützender Diskriminierungsmerkmale dar. (Sozialer Status und chronische Erkrankungen werden hier explizit genannt.) (vgl. Senatsverwaltung für Justiz, Verbraucherschutz und Antidiskriminierung Berlin). 


\section{Zielsetzung}

Hauptziel der Schulungsreihe ist es, die Kompetenzen der Mitarbeitenden im Umgang mit Vielfalt zu erweitern, das Thema in den unterschiedlichen Arbeitsbereichen der Bibliothek weiterzutragen sowie nachhaltig zu verankern und so einen Diversity-Prozess in Gang zu bringen. Konkret bedeutet dies:

- Vielfalt als chancenreiches Zukunftsthema in der Stadtbibliothek thematisieren und aufgreifen,

- potenzielle strukturelle Ausschlüsse in der Stadtbibliothek identifizieren und vermindern,

- das Bewusstsein für Diskriminierungsmechanismen und die Entstehung und Wirkung von Vorurteilen auf individueller, organisationaler und gesellschaftlicher Ebene sensibilisieren und schärfen,

- sich mit der eigenen Wahrnehmung und den eigenen Werten und Verhaltensweisen in Bezug auf Vielfalt auseinandersetzen,

- Handlungskompetenz im Umgang mit kultureller Vielfalt im beruflichen sowie sozialen Alltag erweitern,

- Handlungsalternativen für den Umgang mit schwierigen Situationen erlernen.

Die Planung, Konzipierung und Durchführung der Schulungsreihe sollte durch eine externe Organisation bzw. Agentur mit einschlägiger Fachkompetenz in enger Absprache mit der Stadtbibliothek erfolgen.

\section{Die durchführende Organisation}

\section{Eine Welt der Vielfalt ist}

eine Bildungs- und Beratungsorganisation, die sich seit 1996 aktiv für die Gestaltung einer Gesellschaft der Gleichbehandlung einsetzt. Der Name des Vereins weist auf sein Programm: Eine Welt der Vielfalt ist zugleich ein Bildungsprogramm für Diversity und Antidiskriminierung. Der praxis- und handlungsorientierte Diversity- und Anti-Bias- Ansatz von Eine Welt der Vielfalt basiert auf der Allgemeinen Erklärung der Menschenrechte. (EWdV, Über uns)

Die Organisation arbeitet mit vielen freiberuflichen Trainer*innen. Die Trainings und Fortbildungen werden grundsätzlich in Tandems, also von zwei Trainer*innen, geleitet ${ }^{7}$. Da das Team von Eine Welt der Vielfalt sehr divers aufge-

7 Grundsätzlich begleitet das einmal gewählte Tandem durch die gesamte Fortbildung(sreihe). 
stellt ist und die Trainer`innen unterschiedliche Qualifikationen, Erfahrungen und Schwerpunkte haben, kann für jede Anforderung das passende Team gefunden werden.

\section{Konzept und Aufbau}

Im Rahmen der Schulungsreihe wurden 30 Mitarbeitende zu Diversity-Multiplikator`innen qualifiziert. Der Kreis der Teilnehmenden sollte sich aus Vertre-

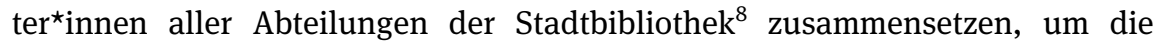
größtmögliche Reichweite zu erzielen. Die Abteilungen waren somit gefragt, jeweils eine Person sowie eine Stellvertretung aus ihrem Team auszuwählen, die an der Schulung regelmäßig teilnehmen soll. Den Entscheidungsprozess durfte jedes Team selbst gestalten.

Das Konzept der Schulungsreihe umfasste drei Bausteine (vgl. Abb. 14), die von den zwei Trainer^innen mit folgenden Unterzielen durchgeführt wurden:
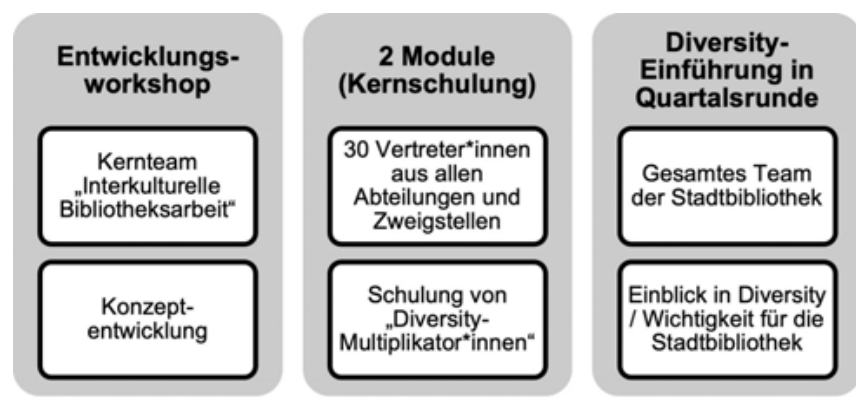

Abb. 14: Bausteine der Schulungsreihe

Die Bausteine im Einzelnen waren:

- Ein halbtägiger Diversity-Entwicklungs-Workshop mit dem interkulturellen Expertinnenteam der Stadtbibliothek Köln und der Bibliotheksleitung.

8 Vertreten waren folgende Abteilungen, Zweigstellen und Teams: Digitale Dienste und Schulservice, Bibliothekarische Systemanalyse und EDV, Bibliothekskundenservice, Interne Kommunikation und Öffentlichkeitsarbeit, Zentrale Bibliotheksverwaltung, Bestandsaufbau und -erschließung, Ausbildungsleitung, alle sieben Sachgebiete der Zentralbibliothek, alle elf Stadtteilbibliotheken, die Germania Judaica und das Kernteam Interkulturelle Bibliotheksarbeit. 
- Diversity-Module zur Schulung von Diversity-Multiplikator`innen: Dabei wurden 30 Mitarbeitende als Vertreter*innen aller internen Abteilungen, Stadtteilbibliotheken und Teams (ausgenommen die Leitungsebene) in zwei Gruppen geschult. Die Module gliederten sich in zwei Teile: ein Grundlagen- und ein Aufbautraining.

- Eine eineinhalbstündige praxisorientierte „Diversity-Einführung“ für alle Mitarbeitenden, die im Rahmen einer der vierteljährlichen Treffen aller Mitarbeitenden, den sogenannten „Quartalsrunden“, stattfinden sollte.

\section{Inhaltliche Gestaltung}

Genauso wichtig wie der konzeptionelle Aufbau waren auch die zu vermittelnden Inhalte der jeweiligen Schulungsbausteine.

\section{Der Entwicklungs-Workshop}

In diesem Baustein wurde die inhaltliche Gestaltung sowie der Ablauf der Trainingsmodule besprochen und an die Bedürfnisse der Bibliotheksmitarbeitenden angepasst. Dafür wurden zunächst die Erwartungen der am Prozess Beteiligten an die Schulung geklärt. So sollte deutlich werden, wo die Bibliothek und ihre Mitarbeitenden bezüglich Vielfalt im Moment stehen, was sie in diesem Bereich noch brauchen und wo sie mit der Schulung hinwollen. Gleichzeitig wurden schon erste Gedanken über die mögliche Rolle der Diversity-Multiplikator`innen gesammelt und zusammengetragen, damit die Trainer`innen die Workshops dementsprechend ausrichten können. Darüber hinaus wurden die Inhalte und Methoden der Schulung vorgestellt. Dabei sollte auch über die Rolle und eine mögliche Einbindung der Leitungsebene in die Schulung nachgedacht werden. Außerdem wurden erste Ideen für die inhaltliche und methodische Gestaltung der Diversity-Einführung im Rahmen der Quartalsrunde für alle Mitarbeitenden gesammelt.

\section{Die Module (Grundlagen- und Aufbautraining)}

Den Kern der Schulungsreihen stellten die Module zur Schulung der DiversityMultiplikator^innen dar. Den geschulten Mitarbeitenden kam dabei eine wichtige aktive Rolle zu: Im Rahmen der Trainings lernten sie, ihr eigenes Arbeits- 
feld $\mathrm{zu}$ analysieren, mit konkreten Methoden den „Diversity-Blick“ in ihre Teams hineinzutragen, Handlungsstrategien für den Umgang mit Vielfalt in der alltäglichen Arbeit zu entwickeln und zu erproben. Mit ihren in den Schulungen erlernten Fähigkeiten sollten sie das Kompetenzteam „Diversity“ bilden, sich untereinander vernetzen und andere Mitarbeitende bei diversitätsorientierten Fragestellungen beraten und unterstützen.

Im Grundlagentraining fand in erster Linie eine Sensibilisierung und Auseinandersetzung auf der individuellen Ebene statt. Dies sollte mit einer guten Mischung aus theoretischem Input und praktischen (Einzel-, Kleingruppenund Großgruppen-)Übungen erreicht werden. Eine besondere Bedeutung kam bei diesen Übungen den anschließenden Reflexionen zu, um die durchgeführten Übungen verarbeiten und einordnen zu können. Im Vordergrund stand eine Auseinandersetzung mit folgenden Themen:

- Wahrnehmungen,

- Stereotype und Vorurteile,

- Identitäten und Empathie,

- Perspektiverweiterung,

- Privilegien,

- individuelle Diskriminierung.

Anhand dieser Themen wurden die Ressourcen der Stadtbibliothek herausgearbeitet, Grundlagen für die Zusammenarbeit festgelegt, Übungen zur Selbstreflexion gemacht, wurde sich mit der eigenen Identität beschäftigt und es wurden andere Identitäten „angenommen“, neue Perspektiven kennengelernt sowie über Handlungsorientierung bzw. Handlungsoptionen diskutiert. Die Themen Identität und Perspektivwechsel spielten eine zentrale und entscheidende Rolle für den Diversity-Prozess und waren daher auch bestimmend an diesen ersten beiden Tagen. Am Ende des Grundlagentrainings sollte eine erste Übertragung von der individuellen auf die organisationale Ebene angestrebt und dabei einige Überlegungen für eine praktische Umsetzung im Bibliotheksalltag gesammelt werden.

Im Aufbautraining ging es vor allem um den Transfer von der individuellen und theoretischen Ebene auf die praktische, organisationsbezogene Arbeit. Thematische Schwerpunkte in diesem Teil waren:

- diskriminierungskritische Sprache,

- strukturelle Diskriminierung und Diskriminierungsmechanismen,

- Machtverhältnisse,

- Rolle der Multiplikator*innen,

- Transfer und Handlungsstrategien. 
Darüber hinaus wurden konkrete Ressourcen der Bibliothek und der Mitarbeitenden, potenzielle Herausforderungen sowie Verbesserungs- und Unterstützungsmöglichkeiten identifiziert. Kern des Aufbautrainings sollte jedoch die Entwicklung von Strategien zum Umgang mit Vielfalt und Diskriminierung sein, die im Arbeitsalltag umsetzbar sind.

\section{Die Diversity-Einführung für alle Mitarbeitenden}

Das gesamte Team der Stadtbibliothek sollte im Rahmen einer Quartalsrunde an einer Diversity-Einführung teilnehmen. In eineinhalb Stunden sollten die Trainer`innen einen kurzen theoretischen Input zum Thema Diversity geben und eine für Großgruppen passende praktische Übung durchführen, deren Ergebnisse im Anschluss gemeinsam ausgewertet und diskutiert werden sollten. Dadurch sollten alle Mitarbeitenden einen praktischen Einblick in das Thema Diversity erhalten und die Bedeutung und die Wichtigkeit des Themas für die Stadtbibliothek erkennen.

\section{Umsetzung und Zwischenbilanz}

Alle Bausteine der Schulung wurden auf einen Zeitraum von vier Monaten (Dezember 2019 bis März 2020) verteilt und terminiert. Um eine konzentrierte und ungestörte Arbeitsatmosphäre fernab des Bibliotheksalltags zu schaffen, fanden die Trainings in externen Räumlichkeiten statt: Als sehr geeignet erwies sich das Zukunftslabor ${ }^{9}$, welches die Stadt Köln städtischen Einrichtungen kostenlos zur Verfügung stellt und sich in unmittelbarer Nähe der Stadtbibliothek befindet.

Das Grundlagentraining kam bei beiden Gruppen sehr gut an. Nicht nur die Inhalte der Schulung, die praktischen Übungen und die großzügigen Reflexions- und Austauschmöglichkeiten in der Gruppe, sondern auch die angenehme und einfühlsame Begleitung durch die beiden Trainer*innen wurden sehr gut bewertet und sorgten für große Motivation, im Prozess zu bleiben und weiter daran zu arbeiten. Auch die Räumlichkeiten trugen zu einer positiven und entspannten Atmosphäre bei und wurden als wichtiger Faktor für das erfolgreiche und unbeschwerte Arbeiten genannt (vor allem aufgrund der dadurch ermög-

9 Das Zukunftslabor ist ein Raum, der Mitarbeitende der Kölner Verwaltung bei der digitalen Transformation und bei der Ideenfindung für die Gestaltung der Zukunft der Stadt unterstützen soll. Mehr dazu unter: https://www.stadt-koeln.de/artikel/69756/index.html. 
lichten räumlichen Trennung vom Arbeitsort und -alltag). Eine Teilnehmerin gab folgendes Feedback:

Die Diversity-Schulung hat mich dazu gebracht, noch einmal über eigene Vorurteile und Vorannahmen nachzudenken. Ich finde es gut, über sich selbst und seine verschiedenen Identitätsanteile, Zugehörigkeiten und Rollen nachzudenken. Was hat mich geprägt? Wo stehe ich in der Gesellschaft? Wie schaue ich auf die Welt? Manche meiner Privilegien waren mir vor der Schulung nicht bewusst. Wo wäre ich heute, ohne sie gehabt zu haben?

Das erste Aufbautraining fand wie geplant Anfang März 2020 noch unter normalen Bedingungen statt. Die in derselben Woche geplante Quartalsrunde mit der Diversity-Einführung für alle Mitarbeitenden musste bereits aufgrund des kurzfristig verhängten Versammlungsverbots für große Gruppen durch die Corona-Lage ausfallen. Das für die darauffolgende Woche geplante zweite Aufbautraining musste schließlich ganz abgesagt werden. Dadurch wurde nicht nur der gerade angelaufene, aber schon sehr erfolgreiche Diversity-Prozess an einem entscheidenden Punkt unterbrochen und in seinem Ablauf verzögert, sondern es entstand ein Ungleichgewicht, da eine Gruppe die Schulung nur zur Hälfte machen konnte. Dadurch war eine Zusammenarbeit beider Gruppen als Kompetenzteam Diversity zunächst nicht möglich.

Ein Nachholversuch für das ausgefallene Aufbautraining in Präsenzform wurde daraufhin organisiert. In der Zwischenzeit wurden einige kleine interne Online-Fortbildungsangebote entwickelt und durchgeführt. Dabei ging es einerseits darum, das Wissen der Schulungsteilnehmenden zu „reaktivieren“, um den Diversity-Prozess aufrechtzuerhalten, andererseits aber auch das gesamte Team der Stadtbibliothek in den Diversity-Prozess einzubinden und zu ermutigen, sich über verschiedene diversitätsorientierte Themen eine Meinung zu bilden und miteinander ins Gespräch zu kommen. So wurde von Teilnehmenden der Schulung eine Diversity-Einführung für alle Mitarbeitenden als Online-Veranstaltung entwickelt und durchgeführt ${ }^{10}$, die einen Einblick sowohl in das Thema Diversity als auch in die Inhalte der Schulung ermöglichte. Zusätzlich wurde anlässlich der Einführung der Kategorie „divers“ im Bibliothekssystem eine interne Online-Informationsveranstaltung zum Thema Geschlechtsoption „divers“ und gendergerechte Sprache durchgeführt.

Zudem stellte sich seit Beginn der Diversity-Schulung heraus, dass die Beratung und die Begleitung durch die Trainer`innen in Form regelmäßiger (digitaler) Austausch- und Reflexionsgespräche - auch über die schon stattgefundenen Trainings hinaus - einen wesentlichen Bestandteil des Diversity-Prozesses

10 Die geplante Einführung mit den Trainerinnen im Rahmen einer Quartalsrunde steht noch aus und wird nachgeholt, sobald es die Umstände erlauben. 
darstellen und von großer Relevanz für dessen nachhaltige Etablierung im Bibliotheksalltag sind. Aus diesem Grund wurde eine zusätzliche Begleitung und Beratung im Rahmen des Diversity-Prozesses für den Zeitraum August bis Dezember 2020 etabliert.

Der Nachholtermin für das Aufbautraining musste jedoch aufgrund der wieder verschärften Corona-Situation mit Versammlungsverbot und Reiseeinschränkungen erneut abgesagt werden. Stattdessen wurde jeweils ein OnlineCoaching pro Gruppe mit den zwei Trainer^innen organisiert, um den Prozess wieder $\mathrm{zu}$ aktivieren und gemeinsam $\mathrm{zu}$ überlegen, wie die Schulungsteilnehmenden im Bereich Diversity zunächst in den jeweiligen Gruppen weiterarbeiten können. Dabei sollten schon entwickelte Ideen und Versuche für kleine Projekte wieder aufgegriffen und weiterentwickelt werden. So beschäftigte sich eine Gruppe mit „Diversity in Kinderbüchern“, einem Thema, das sich bereits im Rahmen des Aufbautrainings der ersten Gruppe als wichtiges Handlungsfeld herauskristallisiert hatte. Aus der weiteren Beschäftigung mit dem Thema wurde ein Konzept für eine Online-Fortbildung entwickelt, die Ende Dezember 2020 für alle Mitarbeitenden angeboten und von vielen in Anspruch genommen wurde.

Ein neuer Nachholtermin für das ausstehende Präsenztraining soll für das letzte Quartal 2021 geplant werden. Bei der Planung für diesen Termin soll dieses Mal die Alternative einer Online-Veranstaltung direkt mitgedacht werden ${ }^{11}$, falls auch dieser Termin aufgrund der Corona-Bedingungen wieder nicht als Präsenzveranstaltung stattfinden kann.

\section{Fazit und Ausblick}

Diversity als Querschnittsthema strukturell zu verankern, bedarf einer umfassenden strategischen Veränderung sowohl auf personeller als auch auf struktureller Ebene. Dies kann jedoch nicht allein mit einer Diversity-Managerin und vereinzelten Maßnahmen nachhaltig umgesetzt werden, weil es dann für die Mitarbeitenden als „von außen übergestülptes“ Verfahren empfunden werden kann. Nur durch eine gemeinsam getragene Strategie kann dieses Vorhaben erfolgreich sein. Aus diesem Grund kann die Schulung von Diversity-Multiplikator^innen einen strategischen Ansatz darstellen und dazu beitragen, die Diver-

11 Mittlerweile kann die Stadtbibliothek auf erfolgreiche Erfahrungen in der Planung von Online-Veranstaltungen zurückgreifen, um dieses Training umzugestalten. Allerdings stellt eine Online-Version nach wie vor eine Notlösung dar, denn nur mit einer Präsenzveranstaltung ist eine angemessene Zusammenarbeit und Sensibilisierung der Mitarbeitenden wirklich möglich. 
sity-Arbeit innerhalb einer Organisation auf eine größere - und im besten Fall inhaltlich und methodisch gut vorbereitete - Gruppe von Mitarbeitenden zu verteilen, die gemeinsam an einem Strang ziehen und das Thema vorantreiben.

Auch wenn aus dem Kreis der geschulten Teilnehmenden einige konkrete Projektideen entstanden sind und diese zum Teil weiterverfolgt wurden, so zeigte sich, dass die Unterbrechung durch die plötzlich auftretenden Corona-Einschränkungen den Diversity-Prozess stark ausbremsten. Die Teilnehmenden waren einerseits mental und organisatorisch mit der neuen Situation und den damit einhergehenden Anforderungen in Beruf und Alltag beschäftigt, so dass nicht mehr viel Raum und Zeit für das Thema Diversity blieb, und andererseits, weil persönliche Treffen nicht oder nur eingeschränkt möglich waren und dadurch die Zusammenarbeit stark beeinträchtigt wurde.

Damit wurde deutlich, dass die Schulung zu einer ersten Sensibilisierung und erfolgreichen Auseinandersetzung mit dem Thema Diversity beigetragen hat. Dennoch reichte dies nicht aus, um diversitätsorientierte Arbeit so zu etablieren, dass die geschulten Mitarbeitenden trotzt unvorhersehbarer Unterbrechungen und sich verändernder Anforderungen im Bibliotheksalltag weiter am Thema bleiben. Aus diesem Grund erscheint es sinnvoll, regelmäßige DiversitySchulungen für alle Mitarbeitenden so früh und flächendeckend wie möglich anzubieten und diese im besten Fall zu einem festen Bestandteil der Fortbildungsmöglichkeiten zu machen. Denn die Sensibilisierung des Personals stellt eine wichtige Grundlage für die Verankerung diversitätsorientierter Arbeit sowie eines tiefergreifenden Diversity-Prozesses dar.

Zur nachhaltigen Etablierung und Weiterentwicklung des Diversity-Prozesses im Bibliotheksalltag ist deshalb geplant, 2021 weitere Online-Fortbildungsmöglichkeiten zu organisieren, um Kenntnisse aufzufrischen und/oder zu erweitern. Darüber hinaus ist angedacht, ein geeignetes Format zu entwickeln, um weitere interessierte Mitarbeitende, die ihr Wissen um Diversity auf- und ausbauen möchten, auf freiwilliger Basis zu schulen. Zudem soll ein DiversitySteuerungskreis mit interessierten, geschulten Mitarbeitenden gebildet werden, um gemeinsam am Thema weiterzuarbeiten und dieses in der Bibliothek zu verankern. 


\section{Literaturverweise}

Abdul-Hussain, S. \& Hofmann, R. (2013). Geschichte. In Online-Dossier Diversitätsmanagement. https://erwachsenenbildung.at/themen/diversitymanagement/grundlagen/ge schichte.php (10.5.2021).

Antidiskriminierungsstelle des Bundes (2020). Allgemeines Gleichbehandlungsgesetz (AGG). https://www.antidiskriminierungsstelle.de/SharedDocs/Downloads/DE/publikationen/ AGG/agg_gleichbehandlungsgesetz.pdf?_blob=publicationFile (10.5.2021).

Bundesministerium der Justiz und für Verbraucherschutz. Grundgesetz für die Bundesrepublik Deutschland, Artikel 2. https://www.gesetze-im-internet.de/gg/art_3.html (10.5.2021).

Generalversammlung der Vereinten Nationen (1948). Resolution der Generalversammlung 217 A (III). Allgemeine Erklärung der Menschenrechte. https://www.un.org/depts/german/ menschenrechte/aemr.pdf (10.5.2021).

Eine Welt der Vielfalt e. V. Über uns. https://www.ewdv-diversity.de/ueber-uns/ (10.5.2021).

Senatsverwaltung für Justiz, Verbraucherschutz und Antidiskriminierung (2020). Landesantidiskriminierungsgesetz (LADG). In: Gesetz- und Verordnungsblatt für Berlin, 76. Jahrgang, Nr. 29.

Tanyılmaz, T. \& Greve, E. (2018). Vielfalt intersektional verstehen. Ein Wegweiser für diversitätsorientierte Organisationsentwicklung. Die Broschüre ist entstanden im Rahmen des Projektes Vielfaltscheck von DeutschPlus, gefördert durch die Robert Bosch Stiftung. https://www.deutsch-plus.de/wp-content/uploads/2018/01/vielfalt-intersektional-verste hen-barrierefrei.pdf. 
